\title{
Antecedentes da retenção de pessoas em organizações: Uma revisão de literatura ${ }^{1}$
}

Antecedents to people retention in organizations: A literature review Variables para la retención de personas en las organizaciones: Una revisión de literatura

\author{
Andrea Valéria STEIL ${ }^{2}$ \\ Mariana Mateus PENHA \\ Maria Alejandra Maldonado BONILLA \\ Universidade Federal de Santa Catarina, Florianopólis, SC, Brasil
}

Resumo

Retenção de pessoas é a capacidade que determinadas organizações têm de manter grupos específicos de profissionais atuando nelas, de modo que alcancem seus objetivos estratégicos. É considerada uma capacidade estratégica em função de sua relação com construtos organizacionais importantes, como conhecimento, criatividade e desempenho. Do ponto de vista do sujeito, a saída da organização tem sido relacionada a elementos estressores. Este artigo apresenta os resultados de uma revisão de literatura sobre os antecedentes da retenção de pessoas em organizações. Para a coleta e seleção de artigos, foram utilizados procedimentos de revisão sistemática de literatura. Os artigos selecionados foram analisados por meio da categorização conceitual de suas variáveis e resultados. Como resultado, foram identificados quatro antecedentes pessoais, trinta e dois antecedentes ocupacionais, dezoito antecedentes organizacionais, e três antecedentes ambientais da retenção de pessoas, os quais são apresentados em uma rede nomológica. $\mathrm{O}$ artigo analisa as implicações destes resultados para a pesquisa em gestão de pessoas e apresenta uma agenda de pesquisas sobre o construto.

Palavras-chave:

Retenção; medidas da retenção; rotatividade de pessoal.

Abstract People retention is the capacity that certain organizations for keeping specific groups of professionals, so that these organizations achieve their objectives. It is a strategic capacity since it is related to important organizational constructs such as knowledge, creativity, and performance. Leaving the organization is also stressing to the individual. This article presents the results of a literature review on the antecedents to people retention in organizations. Systematic literature review procedures were used to collect articles. Selected articles were analyzed through conceptual categorization of their variables and outcomes. As a result, four individual antecedents, thirty-two occupational antecedents, eighteen organizational antecedents, and three environmental antecedents were identified. These are presented in a nomological network. The article analyzes the implications of these results for research and practice in people management and presents a research agenda on the construct. Keywords:

Retention; retention measures; employee turnover.

Resumen

La retención de personas es la capacidade de determinadas organizaciones para formar y mantener grupos específicos de profesionales, de manera que esas organizaciones alcancen sus objetivos estratégicos. Es considerada una capacidad estratégica en virtud de su relación con constructos organizacionales importantes, como conocimiento, creatividad y desempeño. Desde el punto de vista del sujeto, salir de la organización ha sido relacionado a elementos de estrés. Este artículo presenta los resultados de una revisión de

Artigo produzido com o auxílio do Grupo de Pesquisa Interdisciplinar em Conhecimento, Aprendizagem e Memória Organizacional (KLOM) do PPGEGC/UFSC e do Laboratório de Processos de Aprendizagem, Conhecimento e Gestão em Organizações e Trabalho (LABPOT)/Departamento de Psicologia/UFSC.

2 Endereço para correspondência: Departamento de Psicologia, Centro de Filosofia e Ciências Humanas, Universidade Federal de Santa Catarina, Campus Reitor João David Ferreira Lima, Florianópolis, SC, Brasil 88040-900. E-mail: andreasteil@egc.ufsc.br 
literatura sobre las variables que definen la retención de personas en organizaciones. Para ubicar y seleccionar los artículos se utilizó el procedimiento de revisión sistemática de literatura. El análisis de los artículos seleccionados se realizó por medio de la categorización conceptual de sus variables y resultados. Como resultado se identificaron cuatro variables personales, treinta y dos variables ocupacionales, dieciocho variables organizacionales y tres variables de ambiente organizacional, los cuales son presentados en una red nomológica. El artículo analiza las implicaciones de estos resultados para la investigación a cerca de la gestión de personas y presenta un conjunto de trabajos de investigación sobre el constructo.

Palabras-clave:

Retención; medidas de retención; rotación de personal.

U ma das principais preocupações das organizações atualmente é a retenção das pessoas que contribuem para o alcance dos objetivos organizacionais (Hausknecht, Rodda, \& Howard, 2009; Parise, Cross, \& Davenport, 2006). Do ponto de vista prático, é frustrante e caro quando se efetua um processo de recrutamento e seleção longo, programam-se estratégias de socialização, realizam-se ajustes na equipe para a absorção de novos funcionários, investe-se no trabalhador e ele se desliga da organização (Peterson, 2005). A perda de profissionais já estabelecidos na organização pode ser ainda mais prejudicial. Há estimativas de que a perda de um executivo pode custar milhões de dólares por ano à organização. Somado a este aspecto, uma pesquisa com 5 mil executivos indicou que 46\% deles têm a intenção de permanecer em seus cargos por apenas dois a cinco anos (Parise et al., 2006).

Como resposta a esses desafios práticos, a pesquisa científica tem avançado na compreensão das consequências da perda de profissionais e das variáveis antecedentes à retenção. Com relação ao primeiro ponto, há argumentos teóricos que relacionam a falha em reter profissionais qualificados à diminuição da criatividade e da inovação organizacional (Walker, 2001); a altos custos de recrutamento, seleção e treinamento (Hytter, 2007); a prejuízos ao clima organizacional (Rainey \& Steinbauer, 1999); e à perda de habilidades, conhecimento e experiência acumulados na organização (Hytter, 2007; Kyndt, Dochy, Michielsen, \& Moeyaert, 2009). Também há um conjunto de estudos que se dedicaram à compreensão dos antecedentes da retenção, como a influência do mercado de trabalho (Carrell, 2007); clima organizacional e aprendizagem (Govaerts, Kyndt, Dochy, \& Baert, 2011); satisfação no trabalho (Howell, Brumback, Newman, \& Rizzo, 1968); sexo (Barnes \& Jones, 1974); nível educacional (Royalty, 1998); motivação (Mak \& Sockel, 2001); treinamento e desenvolvimento (Doll, 2000), entre outros.

Para além de estudos pontuais sobre os antecedentes da retenção, são escassos os artigos de revisão sobre o construto. Enquanto há várias revisões de literatura sobre rotatividade (p. ex., Heavey, Holwerda, \& Hausknecht, 2013; Steel \& Lounsbury, 2009), localizou-se apenas uma revisão sobre retenção. Apesar do título da revisão localizada ser Turnover and retention research: A glance at the past, a closer review of the present, and a venture into the future (Holtom, Mitchell, Lee, \& Eberly, 2008), a retenção de profissionais é tomada apenas como justificativa para o estudo das variáveis associadas ao turnover. A referida revisão identifica pesquisas passadas sobre rotatividade e estabelece uma agenda pesquisa para este construto, e a retenção permanece em segundo plano. Em função disso, há um conjunto de questões importantes relacionadas aos antecedentes da retenção que ainda não foram respondidas e que requerem maior compreensão.

Para avançar o conhecimento sobre os antecedentes da retenção, os objetivos desta revisão são identificar e sistematizar as variáveis antecedentes da retenção em organizações, pesquisadas em estudos empíricos quantitativos. Esta revisão de literatura contribui para a literatura sobre retenção de pessoas em organizações em pelo menos três formas. Primeiro, busca-se sintetizar as variáveis antecedentes descritas em artigos empíricos publicados em periódicos nacionais e internacionais indexados entre 1945 e 2014. O foco nos artigos empíricos garante a sistematização de variáveis já analisadas empiricamente em diferentes contextos, distanciando-se dos artigos puramente conceituais ou prescritivos sobre o tema.

A segunda contribuição é a organização das variáveis antecedentes em categorias clássicas em psicologia das organizações, como antecedentes pessoais, antecedentes ocupacionais, antecedentes organizacionais e antecedentes ambientais. A compreensão da influência de antecedentes ambientais em um construto como retenção, por exemplo, pode contribuir tanto para a teorização quanto para a prática (Johns, 2006) em organizações de diversos setores, localizadas em regiões com culturas diferentes e imersas em realidades econômicas distintas. A terceira contribuição deste artigo é a apresentação de uma rede nomológica das variáveis antecedentes à 
retenção de pessoas em organizações, composta pelos antecedentes que apresentaram relações significativas com a retenção em pelo menos dois estudos empíricos. Juntas, estas três contribuições podem ajudar a guiar futuros trabalhos teóricos e empíricos sobre retenção de pessoas em organizações.

\section{Retenção de pessoas em organizações}

Retenção de pessoas é a capacidade que determinadas organizações têm de manter grupos específicos de profissionais atuando nelas (Brown, Fraser, Wong, Muise, \& Cummings, 2013; Donoghue, 2010; Johnson, 2000), de modo que alcancem seus objetivos estratégicos (Frank, Finnegan, \& Taylor, 2004). Johnson (2000) agrega um elemento à definição de retenção de pessoas: a comparação do tempo que as pessoas permanecem na organização com o tempo que pessoas com perfis ou cargos similares permanecem em organizações competidoras. Johnson (2000) define, portanto, retenção de pessoas como a capacidade organizacional para manter os profissionais que a organização deseja por mais tempo do que os seus competidores.

Pesquisas sobre retenção em organizações têm focado em diferentes grupos de pessoas. Estas pessoas (a) ocupam cargos determinados em uma organização, (b) atuam em um setor econômico especifico, (c) ocupam cargos similares em diferentes organizações, e/ou, (d) possuem atributos desejáveis à organização. Como exemplos do primeiro grupo citam-se executivos (Ellig, 2013; Taylor \& Finley, 2010), gerentes de enfermagem (Brown et al., 2013), enfermeiras (Frank, 2013; Hillman \& Foster, 2011); médicos de sistema público de saúde (Howell et al., 1968), profissionais recém contratados em organizações (Carr, Pearson, Vest, \& Boyar, 2006), profissionais de sistemas de informação (Mak \& Sockel, 2001), entre outros. Como exemplos de estudos com profissionais que atuam em organizações de um mesmo setor econômico, destacam-se os realizados na indústria farmacêutica (Anis, Rehman, Rehman, Khan, \& Humayoun, 2011), no setor hoteleiro (Hausknecht et al., 2009) e no setor financeiro (Birt, Wallis, \& Winternitz, 2004). Entre os estudos com profissionais que ocupam cargos similares em diferentes organizações destaca-se o estudo com assistentes administrados realizado por Govaerts et al. (2011).

Por fim, pesquisas sobre retenção têm crescentemente focado sua atenção em profissionais que possuem atributos desejáveis à organização, independentemente de cargos ocupados. Estes atributos estão relacionados à demonstração de (a) conhecimentos, habilidades e atitudes valorizados pela organização (Kyndt et al., 2009); ou de (b) resultados específicos, como produtividade (Sheridan, 1992). As pessoas que demonstram estes atributos têm sido chamadas de talentos (Berger \& Berger, 2010; Goldsmith \& Carter, 2010), talentos técnicos (Rothwell, 2011), profissionais valorosos (Cardy \& Lengnick-Hall, 2011) e profissionais qualificados (Kyndt et al., 2009; Mak \& Sockel, 2001; Upadhyay, 2009). Pesquisas com estes profissionais indicam que os mesmos são difíceis de substituir (Upadhyay, 2009) e pedem mais demissão do que são demitidos (Mak \& Sockel, 2001).

Com relação às medidas de retenção, Yamamoto (2011) demonstra que pesquisas empíricas sobre o construto adotam tanto indicadores objetivos (como tempo do profissional na organização e comportamentos de resignação) quanto indicadores subjetivos (cognições relacionadas à saída da organização). Destaca-se que a retenção de pessoas não é o inverso de rotatividade. Enquanto a retenção representa uma capacidade organizacional para manter funcionários considerados importantes na organização, a rotatividade explicita o percentual de mudança na composição de todos os funcionários de uma organização em um determinado período (Waldman \& Arora, 2004). Retenção de pessoas também é diferente de intenção de permanecer na organização. A intenção de permanecer refere-se ao desejo consciente e deliberado do empregado de querer continuar na empresa em que trabalha (Tett \& Meyer, 1993), e é consequente de atitudes direcionadas a comportamentos (Judge \& Kammeyer-Mueller, 2012).

\section{MÉTODO}

Este artigo utilizou procedimentos de revisão sistemática de literatura para responder à seguinte questão de pesquisa: que variáveis antecedentes à retenção de pessoas têm sido analisadas em pesquisas empíricas quantitativas? A revisão sistemática utiliza como fonte de dados a literatura científica sobre uma determinada área e busca rastrear o conhecimento disponível (Sampaio \& Mancini, 2007). Possui, também, procedimentos sistematizados de coleta e análise de dados, de forma que a pesquisa seja transparente e replicável. Estes procedimentos são detalhados a seguir. 


\section{Estratégias de coleta e seleção dos artigos}

Adotaram-se duas estratégias: uma para artigos nacionais e outra para artigos internacionais. Foram utilizados três passos para a busca de artigos científicos nacionais (Figura 1). Inicialmente pesquisou-se em 17 periódicos que tipicamente publicam pesquisas na área de psicologia organizacional e do trabalho (BorgesAndrade \& Zanelli, 2004): Arquivos Brasileiros de Psicologia; Cadernos de Psicologia Social e do Trabalho; Estudos de Psicologia (Natal); Interações: Estudos e Pesquisas em Psicologia (São Marcos); Psico; PsicoUSF; Psicologia em Revista; Psicologia e Sociedade; Psicologia: Ciência e Profissão; Psicologia: Organizações e Trabalho; Psicologia: Reflexão e Crítica; Psicologia: Teoria e Pesquisa; Revista de Administração de Empresas - RAE; Revista de Administração da Universidade de São Paulo - RAUSP; Revista de Administração Pública - RAP; Revista de Estudos Organizacionais - REO; e Revista Organizações \& Sociedade.

Em seguida, com vistas a ampliar o alcance das buscas, foram rastreados os periódicos de Psicologia e Administração indexados à base SciELO. Com este procedimento foram agregados mais sete periódicos: Brazilian Administration Review - BAR; Cadernos EBAPE.BR; Estudos de Psicologia (Campinas); Psicologia em Estudo; RAE Eletrônica. Revista de Administração Contemporânea - RAC; e Revista de Administração Mackenzie - RAM.

Para a busca nestes 24 periódicos utilizou-se o termo retenção nos campos titulo, resumo e palavras-chave em todos os números das revistas disponíveis até 12 de agosto de 2014. Essa busca recuperou 11 artigos (Figura 1).

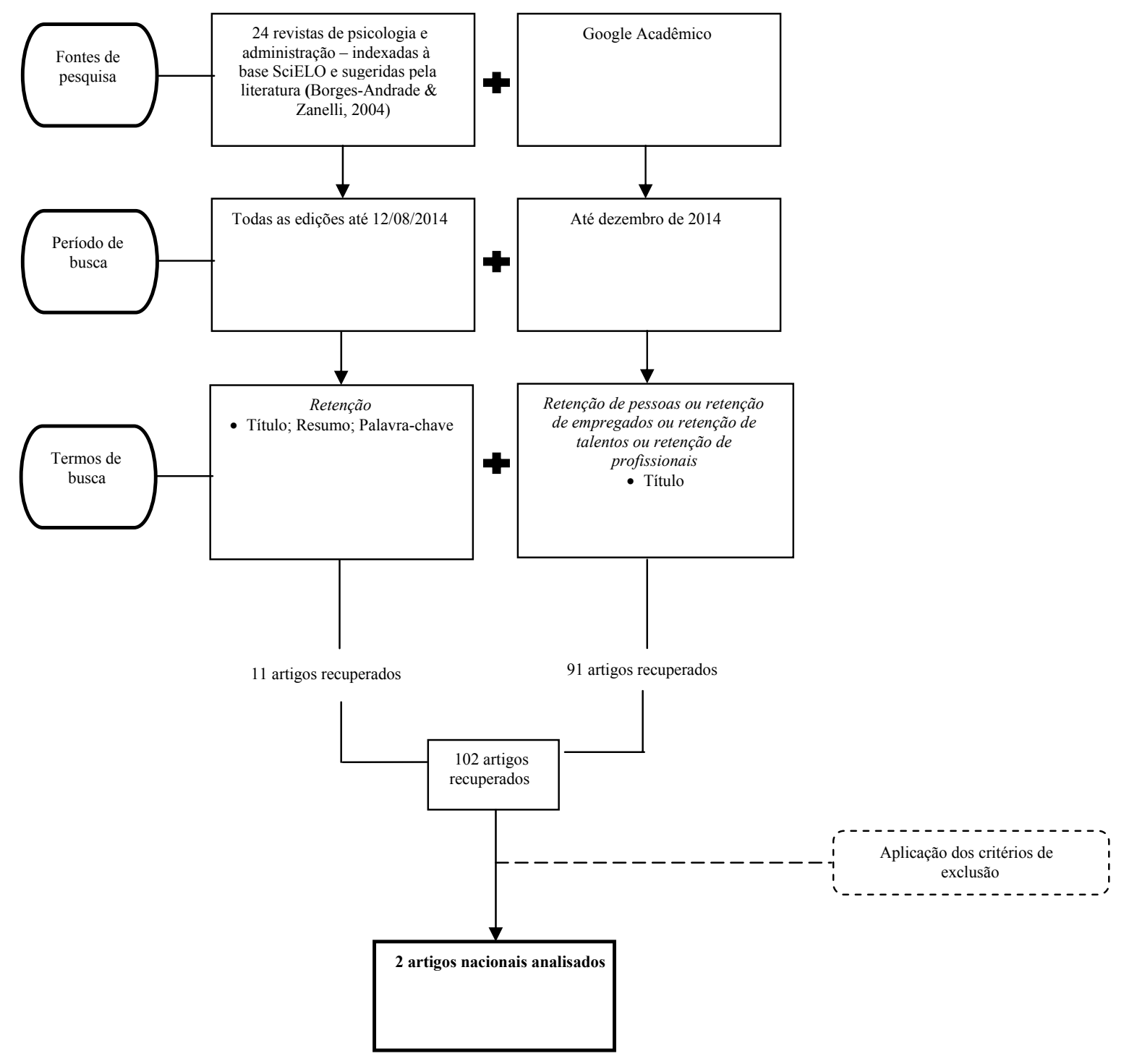

FIGURA 1. Estratégias de busca e seleção de artigos nacionais. 
Para garantir um rastreamento exaustivo da literatura nacional, também foram realizadas buscas no site Google Acadêmico. Neste site foram utilizados quatro termos de busca: retenção de pessoas, ou retenção de empregados, ou retenção de talentos ou retenção de profissionais. Uma vez que o Google Acadêmico só permite a realização de buscas no título dos artigos ou nos textos completos dos mesmos, limitou-se a busca para o campo título, abrangendo todas as publicações até dezembro de 2014. Este procedimento recuperou 91 artigos.

As estratégias de busca de artigos publicados em periódicos nacionais geraram, conjuntamente, 102 artigos (Figura 1). Para se verificar a pertinência da permanência destes 102 artigos na amostra final, foram aplicados critérios explícitos de exclusão, os quais serão apresentados mais a frente.

Os artigos internacionais foram coletados em duas bases de dados: Web of Science e Scopus (Figura 2). Na Web of Science, as buscas foram realizadas em todos os periódicos da sub base Social Sciences Citation Index (SSCI), publicados entre 1945 e 2014. Aplicou-se o termo retention no campo título, resultando em 6.204 publicações. Destas, foram selecionadas apenas aquelas indexadas às áreas de psicologia aplicada (applied psychology) e gestão (management), o que gerou um resultado de 430 publicações mais aderentes à psicologia das organizações. Já na base Scopus, a busca foi realizada inicialmente com o mesmo termo retention na área do conhecimento Social Sciences E Humanities, no período de 1960 a 2014, nos campos título, resumo e palavras-chave. Esta busca gerou 22.750 artigos. Em função da grande quantidade de artigos recuperados, as buscas foram refeitas com os seguintes termos: people retention, ou employee retention, ou talent retention, ou professional retention nos mesmos campos. Com estes termos a busca gerou 279 resultados. No total, as buscas na Web of Science e Scopus somaram 709 artigos.

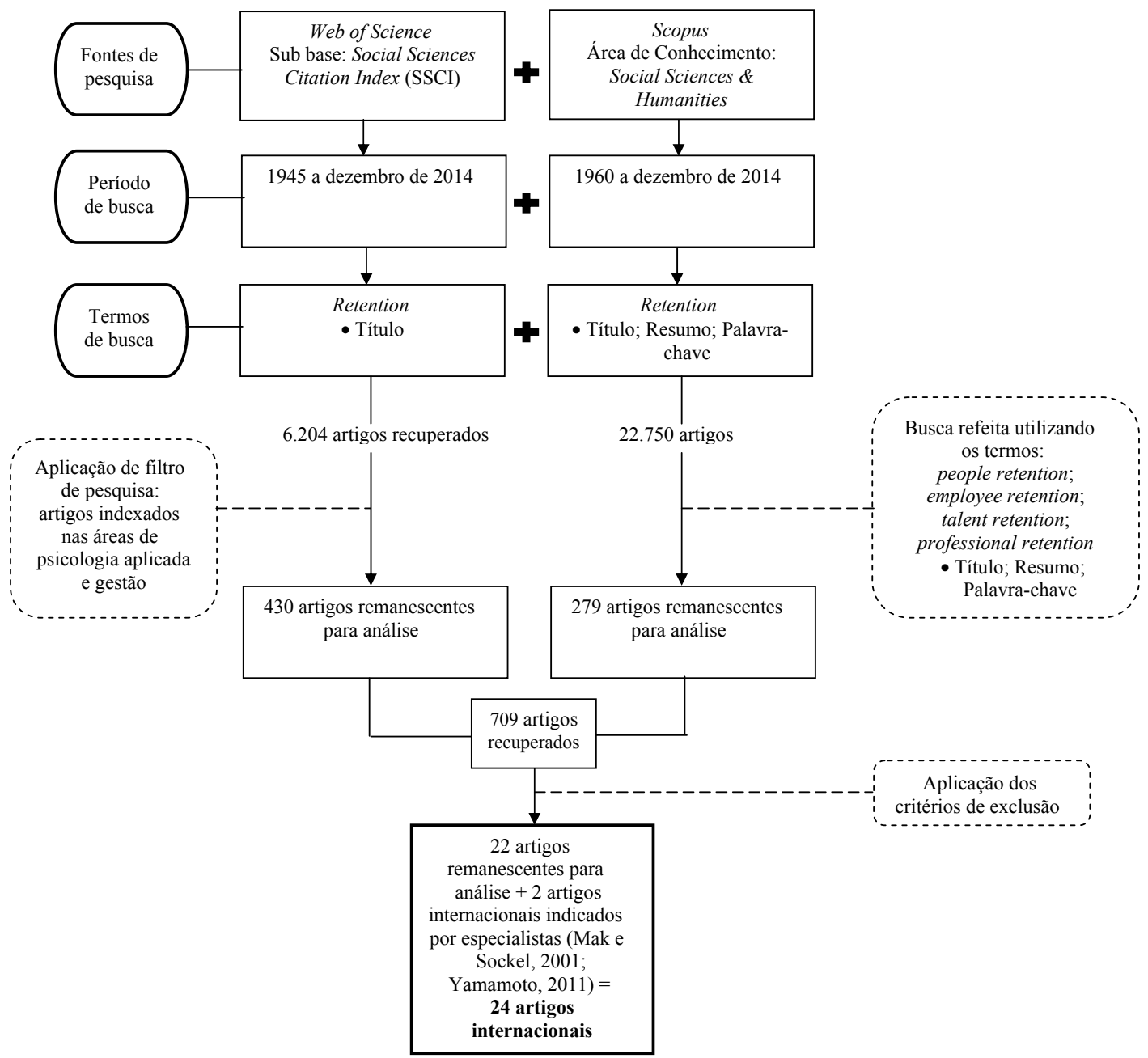

FIGURA 2. Estratégias de busca e seleção de artigos internacionais.

Os resumos dos 811 artigos (soma dos artigos nacionais e internacionais) foram lidos na íntegra, com vistas à aplicação de critérios de exclusão e inclusão. Os seguintes critérios de exclusão foram utilizados, conforme 
a ordem em que se apresentam: (a) artigos sem texto completo na base (5 artigos nacionais e 37 artigos internacionais excluídos); (b) patentes, citações, livros, TCC's, dissertações e teses (47 publicações nacionais e 48 publicações internacionais excluídos); (c) artigos teóricos ou de revisão de literatura (3 artigos nacionais e 24 artigos internacionais excluídos); (d) artigos que utilizam métodos qualitativos (8 artigos nacionais e 46 artigos internacionais excluídos); (e) artigos que não relacionam variáveis empiricamente (3 artigos nacionais e nenhum artigo internacional excluídos); (f) artigos sobre amplas estratégias de retenção sem a identificação de variáveis empíricas (1 artigo nacional e 5 artigos internacionais excluídos); (g) artigos que não abordam o tema retenção de pessoas em organizações (por exemplo, retenção de clientes, de esportistas, de alunos, do conhecimento, da aprendizagem, entre outros) (33 artigos nacionais e 477 artigos internacionais excluídos); (h) artigos que usaram o termo retenção como sinônimo de intenção de permanecer na organização, de intenção de turnover ou como turnover (nenhum artigo nacional e 49 artigos internacionais excluídos); (i) artigos que abordavam apenas variáveis consequentes à retenção (nenhum artigo nacional e 1 artigo internacional excluído). Como critério geral de inclusão, portanto, permaneceram os artigos empíricos que relacionam variáveis antecedentes à retenção de pessoas em organizações. Após a aplicação destes critérios, chegou-se a 2 artigos nacionais e 22 internacionais (Figura 1 e Figura 2).

Conforme previsto em procedimentos de revisão sistemática, e de forma similar a Gosh (2014), Mak e Sockel (2001) e Yamamoto (2011), especialistas na área foram contatatos para que estes indicassem artigos que pudessem não ter sido recuperados a partir das estratégias de busca. Como resposta a este contato, foram incluídos mais 2 artigos internacionais. A amostra final de artigos ficou estabelecida, portanto, em 26 artigos empíricos.

\section{Procedimentos de análise}

Em uma leitura preliminar dos artigos verificaram-se diferenças significativas nos objetivos e nas medidas dos estudos. Em função deste aspecto não foi possível usar procedimentos de meta análise. Alternativamente optou-se pela realização da análise categorial para a interpretação das mensagens contidas nos artigos (Bardin, 2010). Na pré-análise elaborou-se um protocolo com base no objetivo da pesquisa e, para a codificação, foram definidos recortes semânticos.

Não foram determinadas categorias a priori para a agregação das variáveis antecedentes. Dois autores realizaram independentemente a leitura e procederam à codificação e à categorização das variáveis antecedentes. A análise das categorizações individuais indicou uma discordância inicial em cerca de $20 \%$ das categorias (um autor utilizou uma categorização mais específica e outro autor uma mais ampla, por exemplo). Nestes casos optou-se pela utilização de categorias mais amplas, de forma alinhada com a categorização de variáveis utilizada em estudos com construtos relacionados (Werner \& Ward, 2004). Este processo gerou quatro categorias: antecedentes pessoais, antecedentes ocupacionais, antecedentes organizacionais e antecedentes ambientais.

Com a identificação dos antecedentes e suas categorias, procedeu-se ao desenvolvimento da rede nomológica. Uma rede nomológica é um sistema interconectado de leis que relaciona “(a) propriedades observáveis ou quantidades entre si; ou (b) construtos teóricos a construtos observáveis; ou (c) construtos teóricos diferentes entre si” (Cronbach \& Meehl, 1995, p. 290). Uma rede nomológica expressa relações idealizadas, que são progressivamente confirmadas ou desconfirmadas, elaboradas e revisadas com o progresso da ciência (Feigl, 1950).

Como passo necessário à construção da rede nomológica identificaram-se os antecedentes pertencentes a cada categoria, as medidas utilizadas para sua mensuração e a direção da relação identificada. Para ser incluída na rede nomológica, a variável antecedente precisou ter sido testada em dois ou mais estudos e ter apresentado relação similar com a retenção. Com a aplicação deste critérios, oito antecedentes foram identificados para compor a rede nomológica, que será apresentada na próxima seção.

\section{RESULTADOS}

Dos vinte e seis estudos sobre os antecedentes da retenção, a maior proporção destes foi realizada nos Estados Unidos (nove estudos). Os demais foram conduzidos na África do Sul (4 estudos), Paquistão (2 estudos), Europa e Estados Unidos (1 estudo), Taiwan (1 estudo), Japão (1 estudo), Itália (1 estudo), Canadá (1 estudo), Nova Zelândia (1 estudo) e Brasil (1 estudo). Predominaram as pesquisas de levantamento (18 foram levantamentos, 5 foram levantamentos com entrevistas, 1 realizou um quasi-experimento, 1 utilizou modelos 
econômicos e 1 foi estudo de caso), o que era esperado em função da revisão focar os estudos quantitativos. O tamanho das amostras variou entre 70 e 96.554 pessoas.

Para a mensuração do construto retenção, foram utilizadas tanto medidas objetivas quanto escalas de auto-resposta (subjetivas). Dentre as medidas objetivas, predominou o tempo de permanência na organização (11 estudos utilizaram esta medida). Dentre as escalas de auto-resposta, foram usadas as de intenção de sair (5 estudos), de intenção de permanecer (1 estudo) e de percepção de baixa propensão ao turnover (1 estudo). Foram utilizadas também a decisão de permanecer ou de sair da organização (1 estudo), a intenção conjunta de permanecer e de sair (1 estudo) e, por fim, a taxa invertida de rotatividade (1 estudo). Cinco estudos não explicitaram as medidas utilizadas. Ressalta-se que no processo de identificação da amostra de artigos para a realização desta revisão, excluíram-se os artigos que utilizaram o termo retenção como sinônimo de intenção de permanecer, intenção de turnover e turnover. Mesmo assim, na etapa de análise, verificou-se que alguns dos artigos que tratam do construto retenção como capacidade organizacional utilizaram escalas de auto resposta para mensurar a retenção. Com relação a este aspecto, pondera-se que a falta de consenso nas medidas, em pesquisa científica, geralmente está associada à complexidade e à relativa infância do construto (Lähteenmäki, Toivonen, \& Mattila, 2001), características relacionadas ao construto retenção de pessoas.

Embora haja estudos que indiquem a existência de alta correlação entre medidas objetivas e escalas de auto-resposta em alguns construtos (Chandler \& Hanks, 1993), não há uma pesquisa específica que tenha realizado esta avaliação para o construto retenção. Com base em resultados de pesquisas anteriores, o que se sabe é que a intenção de permanecer prediz a retenção e a intenção de sair prediz a rotatividade (Cho, Johanson, \& Guchait, 2009; Igharia \& Greenhaus, 1992). Esta constatação pode indicar que as escalas de auto resposta, utilizadas em pesquisas sobre retenção, estejam mensurando outros construtos, especialmente as cognições relacionadas à permanência ou à saída da organização, e não a retenção per se.

Nos 26 artigos analisados, foram identificadas 57 variáveis antecedentes com relações significativas com a retenção. A Tabela 1 apresenta estas variáveis categorizadas em antecedentes pessoais, ocupacionais, organizacionais e ambientais. Apresenta, também, informações sobre o percentual de artigos que investigou antecedentes por categoria, a direção da relação dos antecedentes com a retenção e os autores de cada estudo.

Os antecedentes pessoais descrevem atributos individuais demográficos. Nesta categoria, 4 antecedentes pessoais foram analisados em 4 estudos empíricos. Os antecedentes ocupacionais referem-se a fatores relacionados ao trabalho realizado. Trinta e dois antecedentes ocupacionais foram associados à retenção de pessoas em 19 estudos. 
TABELA 1. Síntese dos antecedentes da retenção de pessoas, identificados em estudos empíricos sobre o tema

\begin{tabular}{lll}
\hline \multicolumn{1}{c}{ Antecedentes pessoais } & Direção da relação com a retenção & Estudos \\
\multicolumn{1}{c}{ Analisados em 23\% dos artigos } & Positiva & Fottler (1978), Govaerts et al. (2011) \\
Idade & Ser casado (a): positiva & Huang, Lin e Chuang (2006) \\
Estado civil & Mulheres: negativa & Sheridan (1992) \\
Sexo & Negativa & Fottler (1978)
\end{tabular}

$\begin{array}{ll}\text { Antecedentes ocupacionais } & \text { Direção da relação com a retenção } \\ \text { Analisados em } 73 \% \text { dos artigos } & \text { Positiva } \\ \text { Satisfação } & \text { P }\end{array}$

$\begin{array}{ll}\text { Motivação } & \text { Positiva } \\ \text { Lealdade } & \text { Positiva } \\ \text { Ideologia de troca } & \text { Positiva } \\ \text { Salário } & \text { Salário superior: positiva }\end{array}$

Empregado com distinção

Senso de pertencimento

Retribuição variável por desempenho

Trabalho desafiador ou interessante

Liberdade para pensamento inovador

Segurança no trabalho

Metas de desempenho

Bônus por desempenho/comissão

Benefícios para aposentadoria/pensão

Comprometimento organizacional

Práticas de saúde laboral

Existência de representação dos interesses dos funcionários

Turnos de trabalho

Competência em TI

Envolvimento no trabalho

Uso da imaginação no trabalho

Recompensas

Variedade de tarefas

Enriquecimento do trabalho

Liberdade de julgamento no trabalho

Carreira na organização

Liderança

Desempenho

Burnout

Intenção de turnover

Rapidez nas promoções

Sentimento de isolamento
Sim: positiva

Positiva em org. privadas e públicas

Positiva em org. privadas, não nas públicas

Positiva em org. públicas e privadas

Positiva em org. privadas e públicas

Positiva em org. privadas e públicas

Positiva em org. privadas, não nas públicas

Positiva em org. privadas, não nas públicas

Positiva em org. públicas, não nas privadas

Positiva

Positiva

Positiva

Positiva: trabalho no turno do dia

Positiva

Positiva

Positiva

Positiva

Positiva

Positiva

Positiva

Positiva

Positiva

Funcionários com baixo desempenho em culturas com valores relacionados à tarefa: negativa

Negativa

Negativa

Negativa

Negativa em mulheres

\section{Estudos}

Anis et al. (2011), Howell et al. (1968),

Khan, Rehman, Rehman, Safwan e Ahmad. (2011), Terera e Ngirande (2014), Yamamoto (2011)

Mak e Sockel (2001), Mgedezi, Toga e Mjoli (2014)

Mak e Sockel (2001)

Andrews, Witt e Kacmar (2003)

Carrara, Nunes e Sarsur (2014), Carrell (2007), Huang et al. (2006), Oliveira, Ckagnazaroff, Silveira e Moraes (2012), Samuel e Chipunza (2009)

Huang et al. (2006)

Samuel e Chipunza (2009)

Samuel e Chipunza (2009)

Samuel e Chipunza (2009)

Samuel e Chipunza (2009)

Samuel e Chipunza (2009)

Samuel e Chipunza (2009)

Samuel e Chipunza (2009)

Samuel e Chipunza (2009)

Anis et al. (2011)

Oliveira et al. (2012)

Oliveira et al. (2012)

Martin, Sinclair, Lelchook, Wittmer e Charles (2012)

Hair e White (2013)

Mgedezi et al. (2014)

Hlanganipai e Mazanai (2014)

Tererra e Ngirande (2014)

Hlanganipai e Mazanai (2014)

Hlanganipai e Mazanai (2014)

Hlanganipai e Mazanai (2014)

Carrara et al. (2014)

Carrara et al. (2014)

Sheridan (1992)

Mak e Sockel (2001)

Mak e Sockel (2001)

Huang et al. (2006)

Servon e Visser (2011) 


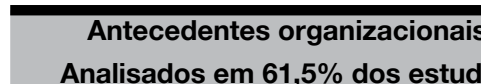

Analisados em $61,5 \%$ dos estudos

Treinamento
Direção da relação com a retenção

Positiva

Positiva

Aumento: positiva

Com valores de relacionamento: positiva (Sheridan, 1992)

Positiva em não gestores (Moncarz et al., 2009)

Promoção

Recrutamento e seleção

Tecnologia de ponta

Creche

Benefícios

Políticas de equilíbrio trabalho/família

Clima de trabalho

Clima de aprendizagem

Capital estrutural

Estabilidade no emprego

Conformidade entre o desempenho de-

mandado e estratégia

Empreendedorismo corporativo

Objetos de $\mathrm{TI}$

Ambiente organizacional interno
Positiva (Moncarz et al., 2009, Oliveira et al. 2012)

Negativa: promoções mais rápidas do que a promoção dos colegas (Huang et al., 2006)

Positiva

Positiva em org. privadas, não nas públicas

Positiva

Positiva

Positiva

Positiva

Positiva

Positiva

Positiva em org. pública

Positiva em org. pública
Estudos

Carrara et al. (2014), Hillman e Foster (2011), Mattox e Jinkeson (2005), Moncarz, Zhao e Kay (2009), Oliveira et al. (2012), Salipante e Goodman (1976), Samuel e Chipunza (2009)

Salipante e Goodman (1976)

Fottler (1978)

Moncarz et al. (2009), Sheridan (1992)

Huang et al. (2006), Moncarz et al. (2009), Oliveira et al. (2012)

Moncarz et al. (2009)

Samuel e Chipunza (2009)

Mann et al. (2011)

Carrara et al. (2014), Yamamoto

(2011)

Yamamoto (2011)

Govaerts et al. (2011)

Govaerts et al. (2011)

Longo e Mura (2011)

Oliveira et al. (2012)

Oliveira et al. (2012)

Haar e White (2013)

Haar e White (2013)

Carrara et al. (2014)

\begin{tabular}{lll}
\hline \multicolumn{1}{c}{ Antecedentes ambientais } & Direção da relação com a retenção & \\
\multicolumn{1}{c}{ Analisados em $\mathbf{1 1 , 5 \%}$ dos estudos } & Recessão: positiva & Huang et al. (2006) \\
Ciclo econômico & Taxa alta: positiva & Carrell (2007) \\
Taxa de desemprego & Sim: relação negativa & Oliveira et al. (2012)
\end{tabular}

Nota. Tabela elaborada pelos autores.

Os antecedentes organizacionais são aqueles relacionados a características e práticas de nível organizacional. Dezoito antecedentes organizacionais foram associados à retenção de pessoas em 16 estudos empíricos. Por fim, os antecedentes ambientais referem-se a estímulos e fenômenos que existem no ambiente externo à organização e que podem influenciar o seu funcionamento. Apenas três antecedentes ambientais foram analisados em 3 estudos.

De modo que novas pesquisas possam ser conduzidas a partir do conhecimento científico acumulado sobre os antecedentes da retenção, desenvolveu-se uma rede nomológica. Como já ressaltado na seção de método, para serem incluídas na rede nomológica, as variáveis antecedentes precisam cumprir o seguinte critério: terem sido testadas em dois ou mais estudos e terem apresentado relações similares com a retenção. Com a aplicação deste critério, oito antecedentes, de três categorias, foram identificados.

$\mathrm{Na}$ categoria dos antecedentes pessoais apenas a idade foi analisada em 2 estudos e em ambos houve relação positiva com a retenção. Nestes estudos, quanto maior a idade, maior a retenção. Dentre os antecedentes ocupacionais, três foram analisados em dois ou mais estudos, todos com relação positiva com a retenção: satisfação (analisada em 5 estudos), motivação (analisada em 2 estudos) e salário (analisado em 5 estudos).

$\mathrm{Na}$ categoria dos antecedentes organizacionais quatro antecedentes foram investigados em dois ou mais estudos: treinamento (investigado em 7 estudos), benefício (investigado em 2 estudos), promoção (investigada em 3 estudos) e cultura (investigada em 2 estudos), todos com relação positiva com a retenção. Por fim, 
nenhum antecedente ambiental foi pesquisado em dois ou mais estudos, por isso não há representante desta categoria na rede nomológica. A rede nomológica pode ser visualizada na Figura 3.

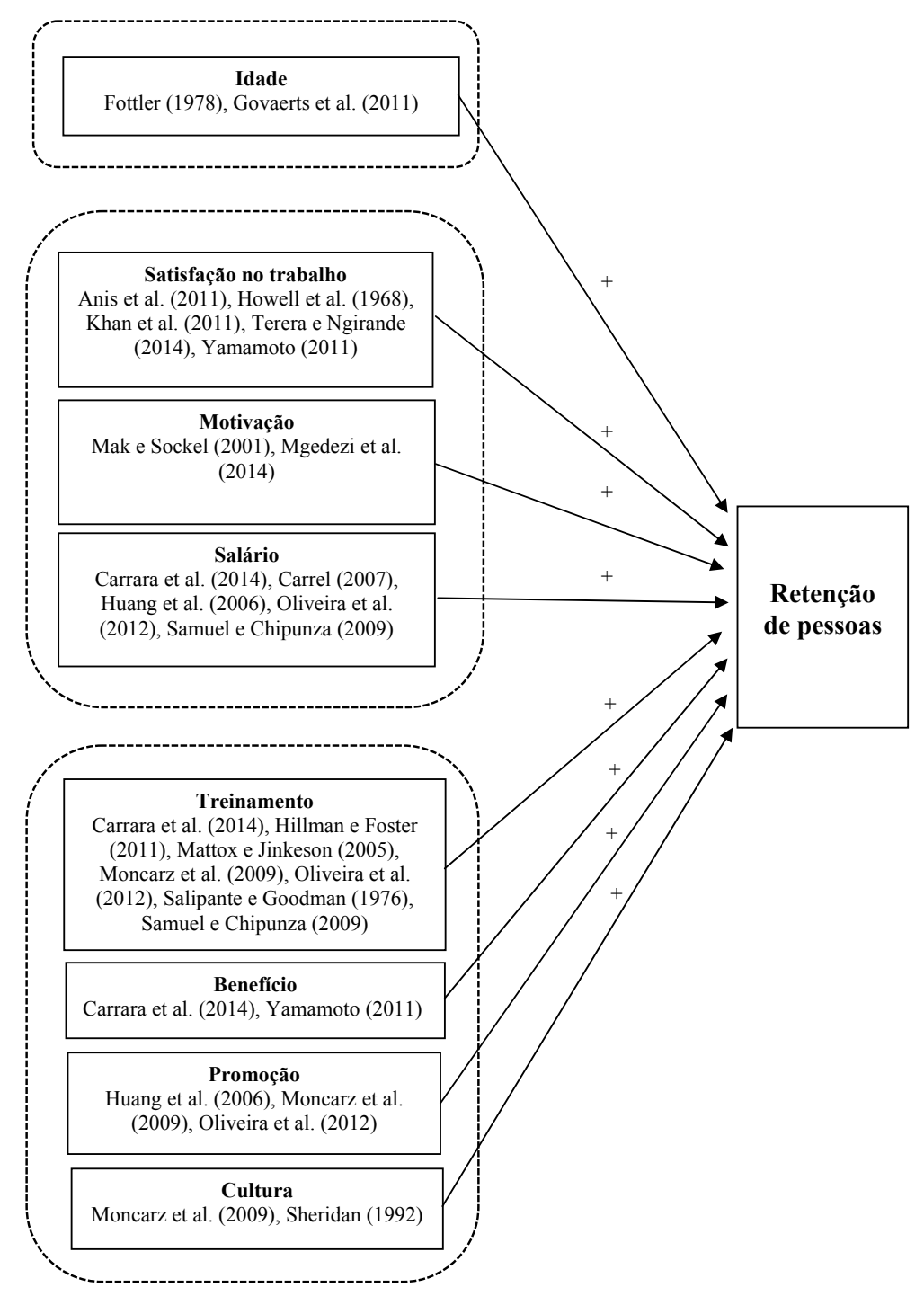

FIGURA 3. Rede nomológica das variáveis antecedentes à retenção de pessoas. Antecedentes pesquisados empiricamente, e com resultados similares, em pelo menos dois estudos.

A rede é apresentada à comunidade científica para estimular a realização de pesquisas empíricas que sejam delineadas a partir do conhecimento prévio acumulado sobre retenção. $\mathrm{O}$ fato das demais 49 variáveis não estarem presentes na rede nomológica não significa que elas não possam ter valor preditivo para a retenção. Significa apenas que não há evidências suficientes, dentro dos critérios estabelecidos nesta revisão, para que elas pertencessem a esta primeira representação das variáveis antecedentes da retenção de pessoas. De fato, a rede nomológica, dentro do proposto por Feigl (1950), representa relações idealizadas, as quais serão progressivamente elaboradas e revisadas a partir da realização de novas pesquisas sobre o tema. Com o avanço das pesquisas sobre os antecedentes da retenção, espera-se que esta rede nomológica seja aperfeiçoada.

Parte dos antecedentes representados na rede nomológica também figuram na nuvem de termos, realizada a partir das 55 palavras-chave dos 26 artigos (foram retiradas as palavras-chave retenção e retenção de funcionários, de modo que a nuvem evidenciasse melhor os antecedentes). $\mathrm{O}$ aplicativo Wordle (usado para a realização da nuvem) destaca as palavras-chave com maior ocorrência (Figura 4). Satisfação no trabalho foi a palavra-chave mais citada (4 vezes), seguida de motivação intrínseca (2 vezes) e gestão de carreira (2 vezes). Tanto satisfação quanto motivação estão presentes na rede nomológica. Além destes antencedentes, a rotatividade também está destacada, tendo sido utilizada como palavra-chave em 3 artigos. 


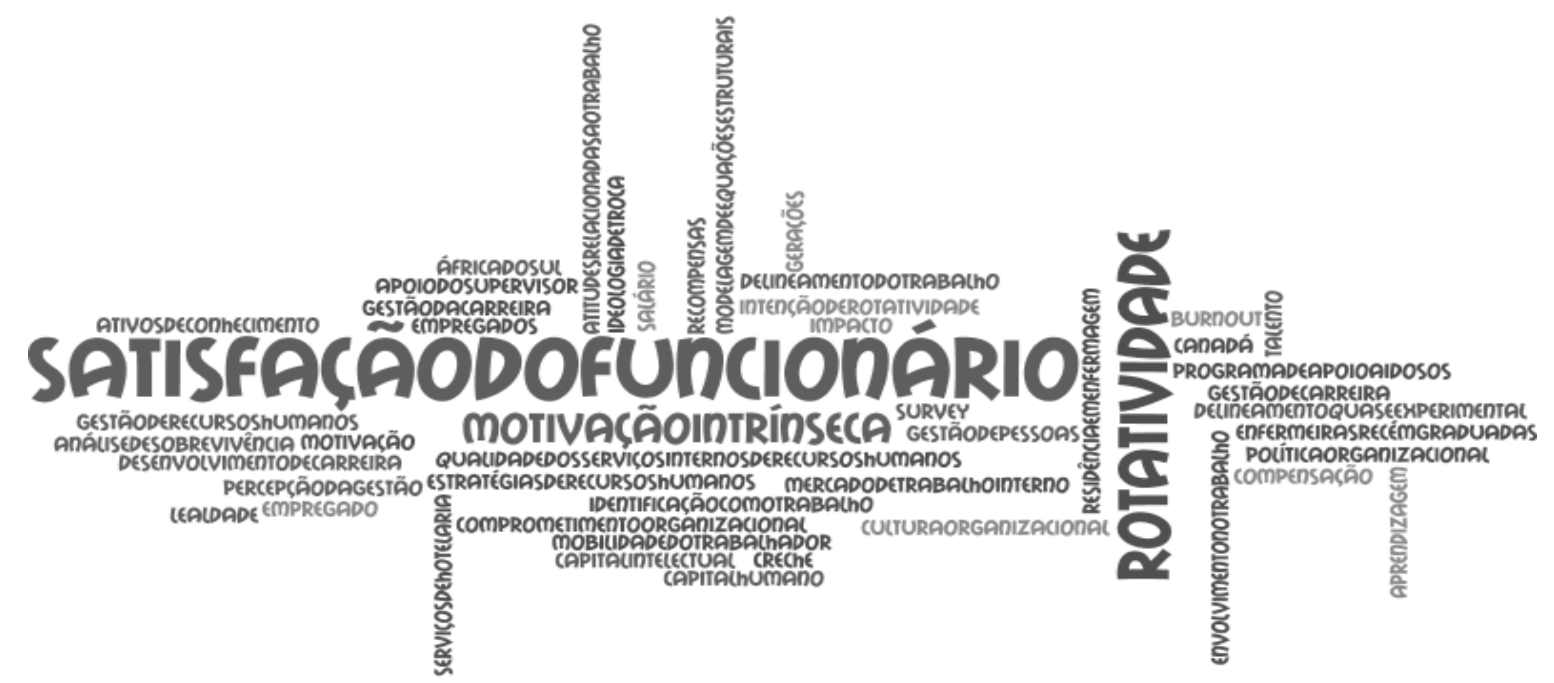

FIGURA 4. Nuvem das palavras-chave utilizadas nos vinte e seis artigos analisados.

Análise complementar pode ser realizada a partir de um estudo da cronologia das publicações sobre antecedentes da retenção. Uma avaliação da distribuição temporal dos estudos demonstra que a grande maioria (84,62\%) foi publicada após os anos 2000, com 7 estudos empíricos entre 2000 e 2010 e 15 estudos a partir de 2011, evidenciando um aumento do interesse científico sobre o tema. A Figura 5 apresesenta uma linha do tempo da quantidade de artigos e de variáveis pesquisadas ao longo do período, organizadas por categorias.

Mais especificamente, na década de 1960 localizou-se 1 estudo sobre os antecedentes da retenção, com foco em uma única variável: a satisfação no trabalho. Na década de 1970 localizaram-se 2 estudos, com cinco variáveis (três organizacionais e duas pessoais). $\mathrm{Na}$ década de 1980 nenhum estudo foi localizado. A década de 1990 apresentou apenas 1 estudo, com uma variável organizacional, uma ocupacional e uma pessoal. Entre 2000 e 20107 estudos foram localizados, com a investigação de duas variáveis ambientais, seis organizacionais, dezesseis ocupacionais e uma pesssoal. Por fim, nos quatro últimos anos (entre 2000 e 2014) 15 estudos foram localizados, com uma variável ambiental, dezessete organizacionais, quatorze ocupacionais e uma pessoal.

\begin{tabular}{|c|c|c|c|c|c|}
\hline $\begin{array}{l}\text { Antecedentes } \\
\text { Ambientais }\end{array}$ & & & & $\begin{array}{c}\text { (2) } \\
\text { ciclo econômico } \\
\text { taxa desemprego }\end{array}$ & $\begin{array}{c}\text { (1) } \\
\text { mudança de governo }\end{array}$ \\
\hline $\begin{array}{l}\text { Antecedentes } \\
\text { Organizacionais }\end{array}$ & & $\begin{array}{c}\text { (3) } \\
\text { treinamento } \\
\text { aconselhamento } \\
\text { tendência aumento vagas }\end{array}$ & $\begin{array}{l}\text { (1) } \\
\text { cultura }\end{array}$ & $\begin{array}{c}(6) \\
\text { treinamento (3) } \\
\ldots\end{array}$ & $\begin{array}{c}(17) \\
\text { treinamento (3) } \\
\ldots\end{array}$ \\
\hline $\begin{array}{l}\text { Antecedentes } \\
\text { Ocupacionais }\end{array}$ & $\begin{array}{c}\text { (1) } \\
\text { satisfação }\end{array}$ & & $\begin{array}{c}\text { (1) } \\
\text { desempenho }\end{array}$ & $\begin{array}{c}(16) \\
\text { salário (3) } \\
\ldots\end{array}$ & $\begin{array}{l}\text { (14) } \\
\text { satisfação (5) } \\
\text { salário (2) } \\
\quad \ldots\end{array}$ \\
\hline \multirow[t]{3}{*}{$\begin{array}{l}\text { Antecedentes } \\
\text { Pessoais }\end{array}$} & & $\begin{array}{c}\text { (2) } \\
\text { idade } \\
\text { nivel educacional }\end{array}$ & $\begin{array}{l}\text { (1) } \\
\text { sexo }\end{array}$ & $\begin{array}{c}\text { (1) } \\
\text { estado civil }\end{array}$ & $\begin{array}{l}\text { (1) } \\
\text { idade }\end{array}$ \\
\hline & 1960-1969 & $1970-1979$ & $1990-1999$ & $2000-2010$ & 2011-2014 \\
\hline & 1 estudo & 2 estudos & 1 estudo & 7 estudos & 15 estudos \\
\hline
\end{tabular}

FIGURA 5. Linha do tempo dos estudos e categorias de antecedentes da retenção de pessoas. Na década de 1980 não foram localizados artigos empíricos sobre antecedentes da retenção. Os números entre parênteses nas categorias significam a quantidade de antecedentes pesquisados naquela categoria por período. Nos períodos com mais do que três antecedentes pesquisados por categoria, são apresentados apenas os antecedentes pesquisados em mais do que um estudo. O número entre parênteses ao lado destes antecedentes significa a quantidade de estudos que investigaram esses antecedentes naquele período. 
Chama a atenção o fato dos antecedentes ambientais serem pesquisados apenas a partir dos anos 2000. Esse movimento parece ser uma tentativa de compreender que variáveis explicam a retenção em um período em que os mercados de trabalho se caracterizam mais fortemente pelas mudanças demográficas, pela falta de pessoas qualificadas no mercado, pelos altos custos de reposição de profissionais qualificados e uma urgência maior, por parte das organizações, de competir por estes profissionais e de mantê-los em seus quadros (Ortlieb \& Sieben, 2012). Um elemento do contexto mundial não pode ser desprezado, a crise econômica e financeira que iniciou em 2007 e está desafiando a forma com que as organizações estão alocando e gerenciando seus ativos, especialmente os ativos intangíveis, com foco nas pessoas (Guevara \& Bounfour, 2013).

\section{DISCUSSÃO}

Esta revisão identificou os antecedentes da retenção de pessoas analisados em estudos empíricos quantitativos e apresentou uma rede nomológica como forma de estimular o desenvolvimento de estudos cumulativos nesta área do conhecimento. Verificou-se que os estudos empíricos têm buscado compreender a capacidade da organização manter os profissionais que deseja atuando nelas por meio, principalmente, da influência de fatores ocupacionais e organizacionais, e, com menor intensidade, dos fatores pessoais e ambientais. Esses resultados indicam que a capacidade para manter profissionais em seus quadros resulta grandemente da estratégia de gestão de pessoas da organização. A estratégia de gestão de pessoas em curso influencia os processos psicossociais que ocorrem na empresa. Entre esses processos destacam-se as atitudes relacionadas ao trabalho, à organização e a comportamentos específicos, incluindo o comportamento de permanecer ou de deixar a organização na qual o indivíduo trabalha (Judge \& Kammeyer-Mueller, 2012).

A partir desta análise, verifica-se que o avanço das pesquisas em retenção de pessoas pode se beneficiar de estudos que aprofundem a compreensão de atitudes relacionadas ao trabalho e à organização, uma vez que elas estão relacionadas com as variáveis ocupacionais identificadas nesta revisão. Exemplos incluem, principalmente, a satisfação no trabalho e o comprometimento organizacional. As atitudes relacionadas a comportamentos também são elementos importantes para a compreensão da retenção, especialmente a intenção de permanecer e a intenção de sair da organização. A meta análise de Steel e Ovalle (1984) demonstrou que a intenção de sair da organização e a saída efetiva da mesma estão relacionadas, sendo a intenção de sair variável preditora da saída efetiva da organização. Estudos que aprofundem esta compreensão no cenário nacional podem contribuir para o entendimento da retenção.

Estudos que investiguem a relação entre as variáveis pessoais e a retenção também são estimulados. Com a diminuição da previsibilidade organizacional e da estabilidade no emprego, as definições tradicionais de carreira linear deixaram de explicar adequadamente a realidade de muitos profissionais, especialmente dos que ingressaram no mercado de trabalho mais recentemente (Steil, 2011). De que forma os antecedentes pessoais influenciam na retenção dos profissionais que vivenciam esta nova realidade de carreira é algo a ser explorado.

É necessário avançar também na compreensão dos efeitos dos ciclos econômicos na retenção de pessoas. Um dos estudos analisados evidenciou que trabalhadores tendem a permanecer mais tempo em seus empregos durante períodos de recessão do que em períodos de prosperidade econômica (Huang et al., 2006). A verificação se esta também é uma característica da realidade brasileira é algo a ser ainda realizado.

Por fim, a literatura sobre retenção de pessos se beneficiaria muito de estudos que analisassem de que forma as diferentes categorias de antecedentes influenciam a retenção de pessoas ao mesmo tempo. $\mathrm{O}$ uso de modelos estruturais com grandes amostras seria um dos caminhos para se avançar nesta compreensão. Apesar de ter sistematizado a literatura nacional e internacional sobre os antecedentes da retenção de pessoas e apresentado uma rede nomológica para estimular novas pesquisas a partir do conhecimento cumulativo sobre o tema, este estudo tem limitações. O estudo não se deteve na investigação mais específica sobre a força (alta, moderada, fraca) das relações identificadas. Entende-se que a compreensão da força destas relações se tornará mais evidente a partir do momento que as pequisas sobre os antecedentes da retenção se avolumem.

\section{REFERÊNCIAS}

Andrews, M. C., Witt, L. A., \& Kacmar, K. M. (2003). The interactive effects of organizational politics and exchange ideology on manager ratings of retention. Journal of Vocational Behavior, 62(2), 357-369. doi:10.1016/S0001-8791(02)00014-3 
Anis, A., Rehman, K., Rehman, I. U., Khan, M. A., \& Humayoun, A. A. (2011). Impact of organizational commitment on job satisfaction and employee retention in pharmaceutical industry. African Journal of Business Management, 5(17), 7316-7324. doi:10.5897/AJBM10.1296

Bardin, L. (2010). Análise de conteúdo. Lisboa: Edições 70.

Barnes, W. F., \& Jones, E. B. (1974). Differences in male and female quitting. Journal of Human Resource, 9(4), 439-451. doi: $10.2307 / 144779$

Berger, L. A., \& Berger, D. R. (2010). The talent management handbook: Creating a sustainable cometitive advantage by selecting, developing, and promoting the best people (2a ed.). New York: MacGraw-Hill.

Birt, M., Wallis, T., \& Winternitz, G. (2004). Talent retention in a changing workplace: An investigation of variables considered important to South African talent. South African Journal Of Business Management, 35(2), 25-31.

Borges-Andrade, J. E., \& Zanelli, J. C. (2004). Psicologia e produção de conhecimento em organizações e trabalho. In J. C. Zanelli, J. E. Borges- Andrade \& A. V. B. Bastos (Orgs.), Psicologia, organizações e trabalho no Brasil (pp. 492-517). Porto Alegre: Artmed.

Brown, P., Fraser, K., Wong, C. A, Muise, M., \& Cummings, G. (2013). Factors influencing intentions to stay and retention of nurse managers: A systematic review. Journal of Nursing Management, 21(3), 459-72. doi:10.1111/j.1365-2834.2012.01352.x

Cardy, R. L., \& Lengnick-Hall, M. L. (2011). Will they stay or will they go? Exploring a customer-oriented approach to employee retention. Journal of Business Psychology, 26(2), 213-217. doi: 10.1007/s10869-011-9223-8

Carr, J. C., Pearson, A. W., Vest, M. J., \& Boyar, S. L. (2006). Prior occupational experience, anticipatory socialization, and employee retention. Journal of Management, 32(3), 343-359. doi: 10.1177/0149206305280749

Carrara, T. M. P., Nunes, S. C., \& Sarsur, A. M. (2014). Fatores de retenção, permanência e desligamento de talentos e de performers. Revista de Administração, Contabilidade e Economia, 13(3), 1119-1148.

Carrell, S. E. (2007). The national internal labor market encounters the local labor market: Effects on employee retention. Labour Economics, 14(2007), 774-787. doi: 10.1016/j.labeco.2006.09.005

Chandler, G. N., \& Hanks, S. H. (1993). Measuring the performance of emerging businesses: A validation study. Journal of Business Venturing, 8(5), 391-408. doi: 10.1016/0883-9026(93)90021-V

Cho, S., Johanson, M. M., \& Guchait, P. (2009). Employees intent to leave: A comparison of determinants of intent to leave versus intent to stay. International Journal of Hospitality Management, 28(3), 374-381. doi:10.1016/j.ijhm.2008.10.007

Cronbach, L. J., \& Meehl, P. E. (1955). Construct validity in psychological tests. Psychological Bulletin, 52(4), 281-302.

Doll, T. M. (2000). View from Washington: Does employer-sponsored training improve retention? Journal of Compensation and Benefits, 16, 35-38.

Donoghue, C. (2010). Nursing home staff turnover and retention: An analysis of national level data. Journal of Applied Gerontology, 29(1), 89-106. doi:10.1177/0733464809334899

Ellig, B. R. (2013). Attracting, motivating and retaining executives: Lessons from years as an HR executive. Compensation \& Benefits Review, 45(2), 75-87. doi: 10.1177/0886368713492119

Feigl, H. (1950). Existencial hyphoteses: Realistic versus phenomenalistic interpretations. Phylosophy of Science, 17(1), 36-62.

Fottler, M. D. (1978). Retention of the hard-core unemployed. Academy of Management Journal, 21(3), 366-79.

Frank, F. D., Finnegan, R. P., \& Taylor, C. R. (2004). The race for talent: Retaining and engaging workers in the 21 st century. Human Resource Planning, 27(3), 12-25.

Frank, B. (2013). Improving nurse faculty retention through a phased retirement process. Journal of Nursing Management, 21(7), 922-926. doi: 10.1111/jonm.12176

Goldsmith, M., \& Carter, L. (2010). Best practices in talent management: How the wold's leading corporations manage, develop, and retain top talent. San Francisco: John Wiley \& Sons.

Gosh, R. (2014). Antecedents of mentoring support: A meta-analysis of individual, relational, and structural or organizational factors. Journal of Vocational Behavior, 84(3), 367-384. doi: 10.1016/j.jvb.2014.02.009

Govaerts, N., Kyndt, E., Dochy, F., \& Baert, H. (2011). Influence of learning and working climate on the retention of talented employees. Journal of Workplace Learning, 23(1), 35-55. doi: http://dx.doi.org/10.1108/13665621111097245

Guevara, D., \& Bounfour, A. (2013). Monitoring intellectual capital: A case study of a large company during the recent economic crisis. Knowledge Management Research \& Practice, 11(2), 196-207. doi: 10.1057/kmrp.2013.12 
Haar, J. M., \& White, B. J. (2013). Corporate entrepreneurship and information technology towards employee retention: A study of New Zealand firms. Human Resource Management Journal, 23(1), 109-125. doi:10.1111/j.1748-8583.2011.00178.x

Hausknecht, J. P., Rodda, J., \& Howard, M. J. (2009). Targeted employee retention: Performance based and job-related differences in reported reasons for staying. Human Resource Management, 48(2), 269- 288.

Heavey, A. L., Holwerda, J. A., \& Hausknecht, J. P. (2013). Causes and consequences of collective turnover: A meta-analytic review. Journal of Applied Psychology, 98(3), 412-453. doi: 10.1037/a0032380.

Hillman, L., \& Foster, R. R. (2011). The impact of a nursing transitions programme on retention and cost savings. Journal of Nursing Management, 19(1), 50-6. doi:10.1111/j.1365-2834.2010.01187.x

Hlanganipai, N., \& Mazanai, M. (2014). Career management practices: Impact of work design on employee retention. Mediterranean Journal of Social Sciences, 5(4), 21-31. doi: 10.5901/mjss.2014.v5n4p21

Holtom, B. C., Mitchell, T. R., Lee, T. W., \& Eberly, M. B. (2008). Turnover and retention research: A glance at the past, a closer review of the present, and a venture into the future. The Academy of Management Annals, 2(1), 231-274. doi: $10.1080 / 19416520802211552$

Howell, M. A., Brumback, G. B., Newman, S. H., \& Rizzo, J. R. (1968). Work satisfaction and the retention of organizationally employed physicians. Personnel Psychology, 21(1), 63-77. doi: 10.1111/j.1744-6570.1968.tb02288.x

Huang, I., Lin, H., \& Chuang, C. H. (2006). Constructing factors related to worker retention. International Journal of Manpower, 27(5), 491-508. doi:10.1108/01437720610683976

Hytter, A. (2007). Retention strategies in France. The Irish Journal of Management, 28(1), 59-80.

Igharia, I., \& Greenhaus, J. (1992). The career advancement prospects of managers and professionals. Decision Sciences, 23(2), 478-500. doi: 10.1111/j.1540-5915.1992.tb00401

Johns, G. (2006). The essential impact of context on organizational behavior. Academy of Management Review, 31(2), 386-408.

Johnson, E. J. (2000). The nursing shortage: From warning to watershed. Applied Nursing Reaserch, 13(3), 162-163.

Judge, T. A., \& Kammeyer-Mueller, J. D. (2012). Job attitudes. Annual Review of Psychology, 63(1), 341-367. doi: 10.1146/ annurev-psych-120710-100511

Khan, M. A., Rehman, K. U., Rehman, I. U., Safwan, N., \& Ahmad, A. (2011). Modeling link between internal service quality in human resources management and employees retention : A case of Pakistani privatized and public sector banks. African Journal of Business Management, 5(3), 949-959. doi:10.5897/AJBM10.829

Kyndt, E., Dochy, F., Michielsen, M., \& Moeyaert, B. (2009). Employee retention: Organisational and personal perspectives. Vocations and Learning, 2(3), 195-215. doi: 10.1007/s12186-009-9024-7

Lähteenmäki, S., Toivonen, J., \& Mattila, M. (2001). Critical aspects of organizational learning research and proposals for its measurement. British Journal of Management, 12(2), 113-129. doi: 10.1111/1467-8551.00189

Longo, M., \& Mura, M. (2011). The effect of intellectual capital on employees' satisfaction and retention. Information \& Management, 48(7), 278-287. doi:10.1016/j.im.2011.06.005

Mak, B. L., \& Sockel, H. (2001). A confirmatory factor analysis of IS employee motivation and retention. Information \& Management, 38(5), 265-276. doi: 10.1016/S0378-7206(00)00055-0

Martin, J. E., Sinclair, R. R., Lelchook, A. M., Wittmer, J. L. S., \& Charles, K. E. (2012). Non-standard work schedules and retention in the entry-level hourly workforce. Journal of Occupational and Organizational Psychology, 85(1), 1-22. doi:10.1348/096317910X526803

Mattox, J. R., \& Jinkerson, D. L. (2005). Using survival analysis to demonstrate the effects of training on employee retention. Evaluation and Program Planning, 28(4), 423-430. doi: 10.1016/j.evalprogplan.2005.07.006

Mgedezi, S., Toga, R., \& Mjoli, T. (2014). Intrinsic motivation and job involvement on employee retention: Case study - A selection of eastern cape government departments. Mediterranean Journal of Social Sciences, 5(20), 2119-2126. doi: 10.5901/ mjss.2014.v5n20p2119

Moncarz, E., Zhao, J., \& Kay, C. (2009). An exploratory study of US lodging properties' organizational practices on employee turnover and retention. International Journal of Contemporary Hospitality Management, 21(4), 437-458. doi:10.1108/09596110910955695

Oliveira, K. P., Ckagnazaroff, I. B., Silveira, M. C., \& Moraes, C. E. S. (2012). Retenção de pessoas no setor público mineiro: O caso dos especialistas em políticas públicas e gestão governamental. Revista de Carreiras e Pessoas, 2(3), 46-64. 
Ortlieb, R. \& Sieben, B. (2012). How to safeguard critical resources of professional and managerial staff: Exploration of a taxonomy or resource retention strategies. The international journal of human resource management, 23(8), 1688-1704. doi: $10.1080 / 09585192.2011 .610341$

Parise, S., Cross, R., \& Davenport, T. (2006). Strategies for preventing a knowledge-loss crisis. MIT Sloan Management Review, $47(4), 31-38$

Peterson, C. H. (2005). Employee retention: The secrets behind Wal-Mart's successful hiring policies. Human Resource Management, 44(1), 85-88. doi: 10.1002/hrm.20044

Rainey, H. G., \& Steinbauer, P. (1999). Galloping elephants: Developing elements of a theory of effective government organizations. Journal of Public Administration Research and Theory, 9(1), 1-32.

Rothwell, W. J. (2011). Invaluable knowledge: Securing your company's technical expertise. New York: AMACOM.

Royalty, A. B. (1998). Job-to-job and job-to-nonemployment turnover by gender and education level. Journal of Labor Economics, 16(3), 392-443. doi: 10.1086/209894

Salipante, P., \& Goodman, P. (1976). Training, counseling, and retention of the hard-core unemployed. Journal of Applied Psychology, 61(1), 1-11.

Sampaio, R., \& Mancini, M. (2007). Estudos de revisão sistemática: Um guia para síntese. Revista Brasileira de Fisioterapia, 11(1), 83-89.

Samuel, M. O., \& Chipunza, C. (2009). Employee retention and turnover: Using motivational variables as a panacea. African Journal of Business Management, 3(8), 410-415. doi:10.5897/AJBM09.125

Servon, L. J., \& Visser, M. A. (2011). Progress hindered: The retention and advancement of women in science, engineering and technology careers. Human Resource Management Journal, 21(3), 272-284. doi:10.1111/j.1748-8583.2010.00152.x

Sheridan, J. E. (1992). Organizational culture and employee retention. Academy of Management Journal, 35(5), $1036-1056$.

Steel, R. P., \& Ovalle, N. K. (1984). A review and meta-analysis of research on the relationship between behavioral intentions and employee turnover. Journal of Applied Psychology, 69(4), 673-686. doi: 10.1037/0021-9010.69.4.673

Steel, R. P., \& Lounsbury, J. W. (2009). Turnover process models: Review and synthesis of a conceptual literature. Human Resource Management Review, 19(4), 271-282. doi: 10.1016/j.hrmr.2009.04.002

Steil, A. V. (2011). Trajetória interdisciplinar formativa e profissional na sociedade do conhecimento. In A. Philippi Jr. \& A. J. Silva Neto (Orgs.), Interdisciplinaridade em ciência, tecnologia e inovação (pp. 209-228). Barueri: Manole.

Taylor, M., \& Finley, D. (2010). Acculturation, assimilation, and retention of international workers in resorts. International Journal of Contemporary Hospitality Management, 22(5), 681-692. doi: http://dx.doi.org/10.1108/09596111011053800

Terera, S. R., \& Ngirande, H. (2014). The impact of rewards on job satisfaction and employee retention. Mediterranean Journal of Social Sciences, 5(1), 481-487. doi: 10.5901/mjss.2014.v5n1p481

Tett, R. P., \& Meyer, J. P. (1993). Job satisfaction, organizational commitment, turnover intention, and turnover: Path analysis based on meta-analytic findings. Personnel Psychology, 46(2), 259-293. doi: 10.1111/j.1744-6570.1993.tb00874.x

Upadhyay, S. S. (2009). Compensation management: Rewarding performance. New Dehli: Global India Publications.

Waldman, J. D., \& Arora, S. (2004). Measuring retention rather than turnover: A different and complementary HR calculus. Human Resource Planning, 27(3), 1-9.

Walker, J. (2001). Zero defections? Human Resource Planning, 24(1), 6-8.

Werner, S., \& Ward, S. G. (2004). Recent compensation research: An eclectic review. Human Resource Management Review, 14(2), 201-227. doi: 10.1016/j.hrmr.2004.05.003

Yamamoto, H. (2011). The relationship between employee benefit management and employee retention. The International Journal of Human Resource Management, 22(17), 3550-3564. doi: 10.1080/09585192.2011.560871 